\title{
Rotinas organizacionais: uma análise bibliométrica
}

\author{
Organizational routines: a bibliometric analysis \\ Rutinas organizacionales: un análisis bibliométrico
}

\section{Resumo}

As rotinas organizacionais se revelam um importante mecanismo na dinâmica de uma organização. O seu estudo tem passado por diferentes visões ao longo do tempo, visto que eram entendidas inicialmente como meros mecanismos de estabilidade e confiabilidade, passando a serem vistas como instrumentos indutores de inovação e mudança. Essa evolução de entendimento ainda ocorre e faz-se necessário entender como o estudo de rotinas organizacionais pode contribuir para o desenvolvimento organizacional. Este estudo tem como objetivo apresentar uma análise bibliométrica sobre rotinas organizacionais, caracterizando o panorama de pesquisa mais recente sobre o tema. Foram pesquisados artigos publicados sobre rotinas organizacionais nas bases de dados Scielo, Scopus e Web of Science para o período de 2015 a 2019, tendo sido analisados e classificados em termos de periódicos, países de origem, palavras-chave, número de citações, temas associados e tipo de estudo. Os resultados apontam que os estudos mais recentes compartilham a visão moderna de que as rotinas organizacionais são hoje um elemento da dinâmica organizacional, permitindo o processo de mudança. A análise bibliométrica, além de apresentar um método confiável e replicável para análise de um determinado campo de estudo, identificou uma escassez de pesquisa sobre o tema no Brasil, indicando que há uma lacuna entre o conhecimento científico produzido nacionalmente e no exterior. A pesquisa indicou, ainda, diversos temas associados como dinâmica das rotinas, inovação, mudança, aprendizagem, capacidades dinâmicas e estabilidade, permitindo a gestores que apliquem em suas organizações novas abordagens para as rotinas organizacionais.

Palavras-chave: Rotinas organizacionais; Análise bibliométrica; Campo de estudo.

\begin{abstract}
The organizational routines reveal themselves as an important mechanism in the orgnizational dynamics. Their study has been through diferent visions over time, since were initially perceived as simple mechanisms of stability and reliability, starting to be seen as inductive instruments of innovation and change. This evolution of understanding still occurs and it's necessary to know how the organizational routines studies may contribute to the organizational develpment. This particular study has the purpose to present a bibliometric analysis about organizational routines, characterizing the most recent panorama about the subject matter. Scientific articles about organizational routines were surveyed at Scielo, Scopus and Web of Science databases in the period from 2015 to 2019, then analysed and labeled by journals, countries of origin, keywords, number of citations, associated themes and study types. The results points out that the most recent studies share the modern view that the organizational routines are an organizational dynamics element, which allows the process of change. The bibliometric analysis, besides of exhibit a reliable and replicable method to analyse a particular field of study, has identified a scarcity of research on this matter in Brazil, showing a gap between the scientific knowledge produced nationally and abroad. The research has also indicated several associated themes like routines dynamics, innovation, change, learning, dynamic capabilities and stability, allowing the managers to apply new approaches to the organizational routines in theirs organizations.
\end{abstract}

Keywords: Organizational routines; Bibliometric analysis; Field of study.

\section{Resumen}

Las rutinas organizacionales son un mecanismo importante en la dinámica de una organización. Su estudio ha pasado por diferentes visiones a lo largo del tiempo, ya que inicialmente se entendieron como meros mecanismos de estabilidad 
y confiabilidad, pasando a ser vistos como instrumentos que inducen la innovación y el cambio. Esta evolución de la comprensión aún ocurre y es necesario comprender cómo el estudio de las rutinas organizacionales puede contribuir al desarrollo organizacional. Este estudio tiene como objetivo presentar un análisis bibliométrico sobre las rutinas organizacionales, caracterizando las investigaciones más recientes sobre el tema. Se buscaron artículos publicados sobre rutinas organizacionales en las bases de datos Scielo, Scopus y Web of Science para el período 2015-2019, habiendo sido analizados y clasificados en cuanto a revistas, países de origen, palabras clave, número de citas, temas asociados y tipo de estudio. Los resultados muestran que los estudios más recientes comparten la visión moderna de que las rutinas organizacionales son hoy un elemento de la dinámica organizacional, permitiendo el proceso de cambio. El análisis bibliométrico, además de presentar un método confiable y replicable para analizar un campo de estudio en particular, identificó una falta de investigación sobre el tema en Brasil, lo que indica que existe una brecha entre el conocimiento científico producido a nivel nacional y en el exterior. La investigación también indicó varios temas asociados como la dinámica de las rutinas, la innovación, el cambio, el aprendizaje, las capacidades dinámicas y la estabilidad, lo que permite a los gerentes aplicar nuevos enfoques a las rutinas organizacionales en sus organizaciones.

Palabras clave: Rutinas organizacionales; Análisis bibliométrico; Campo de estúdio.

\section{Introdução}

Os estudos sobre rotinas organizacionais (RO) têm crescido nos últimos anos em virtude de terem passado por uma importante mudança em seu entendimento no contexto das organizações. Inicialmente, eram vistas como fatores de inércia e impeditivos aos processos de inovação e mudança organizacional. Porém, estudos como os de Feldman e Pentland (2000, 2003, 2008) trouxeram uma nova perspectiva ao tema ao indicarem que as rotinas organizacionais, ao contrário do que afirmavam os estudos anteriores, possuem o condão de induzir mudança

Os atores que estabeleceram a visão tradicional (Cyert \& March, 1963; Gersick \& Hackman, 1990; Nelson \& Winter, 1982) relacionam rotinas com estabilidade e confiabilidade. De acordo com Cyert e March (1963), as organizações desenvolvem as rotinas e estabilizam-nas, e isso as tornam fontes de confiabilidade. Já Nelson e Winter (1982) acreditam que uma vez que o conjunto de rotinas faça parte da memória organizacional em virtude do uso, os planos podem não ser necessários. Nesse mesmo sentido, Gersick e Hackman (1990), explicam que o estabelecimento das rotinas possibilita reduzir a incerteza e aumentar a confiança dos indivíduos dentro do grupo.

Ao analisarem os conceitos formulados pelos estudos tradicionais, Schröder e Geiger (2016) destacam que a visão das rotinas na perspectiva tradicional considera que elas asseguram resultados estáveis e confiáveis na medida em que realizam tarefas recorrentes. Nesta perspectiva, alguns autores como Nascimento, Silva, Oliveira, Nascimento, Gomes \& Moreira (2020), defendem que normas e rotinas podem ser de extrema importância para os profissionais inseridos na área de saúde, padronizando assim, seus procedimentos.

A visão mais recente difere da visão tradicional que associa rotinas à estabilidade, repetição e padrões de comportamentos automáticos (Dittrich, Guérard \& Seidl, 2016). A grande mudança neste cenário, de acordo com Schröder e Geiger (2016), ocorreu a partir do ano 2000, momento que marcou a modificação substancial da compreensão de que as rotinas privilegiam a estabilidade, em razão de um estudo concebido inicialmente para abordar estabilidade ter encontrado evidências de que as rotinas, além disso, são fontes de mudança. O estudo de Feldman (2000) sugere que as rotinas não são necessariamente fixas e automáticas, mas elementos dinâmicos que podem produzir tanto a estabilidade quanto mudança. Esta perspectiva introduz a compreensão de rotinas como fontes de mudança endógena, além de considerar os efeitos da ação do indivíduo, visto que nos estudos anteriores pouca atenção foi dada às ações desenvolvidas pelos atores envolvidos na execução das rotinas (Feldman, Pentland, D’adderio \& Lazaric, 2016). Nesta nova visão, o entendimento das rotinas compreende a reflexão sobre os aspectos que as compõem: o ostensivo, o performativo e os artefatos. O aspecto ostensivo se caracteriza como a forma ideal ou ideia generalizada sobre a rotina; já o performativo é seu viés prático, ou seja, as ações realizadas pelas pessoas em determinado momento e local; e os artefatos são os resumos objetificados das rotinas (Feldman, Pentland, 2003). 
De acordo com esta visão, Feldman e Pentland (2003, p. 96) estabeleceram a definição de rotina organizacional como "um padrão repetitivo e reconhecível de ações interdependentes, envolvendo múltiplos atores". Os autores destacam que o fato de as rotinas serem documentadas através de um conjunto de procedimentos e regras formais não significa que esta forma de documentação necessariamente conste na definição central delas. Para Pentland e Feldman (2005), através do entendimento detalhado das rotinas é possível aplicar diversas teorias das ciências sociais para explicar suas diferenças; além disso, entender sua dinâmica interna é importante se existe o desejo de gerenciá-las.

O desenvolvimento dos estudos sobre rotinas organizacionais possibilitou a compreensão delas através da relação entre a ideia abstrata (estrutura) e o desempenho real dos indivíduos (agência). De acordo com Parmigiani e Howard-Grenville (2011), Feldman e Pentland desenvolveram um conceito muito importante para o estudo das rotinas no artigo "Reconceptualizing organizational routines as a source of flexibility and change" de 2003, na medida em que desenvolveram a distinção entre os aspectos ostensivo e performativo. Concordando com o entendimento dos autores citados, Schröder e Geiger (2016) destacam como elemento essencial para a mudança na dinâmica do estudo sobre rotinas a diferenciação entre os aspectos ostensivo, performativo e material (ou artefatos) das rotinas.

Diante do exposto, a utilização das rotinas como objeto de estudo se justifica pela sua importância no contexto das organizações, pois podem ser consideradas como base dos processos de trabalho que envolvem coordenação entre atores (Pentland \& Feldman, 2008; Melo \& Machado, 2020; Broman, Ruas, \& Rocha-pinto, 2019; Figueira \& Rocha-Pinto, 2018). Diante da relevância no contexto organizacional, Becker (2008) destacou o número crescente de estudos empíricos sobre os fenômenos organizacionais que utilizam as rotinas como meios de análise. Alguns desses estudos voltaram-se mais a visão tradicional sobre rotina enquanto estudos posteriores, influenciados pelos achados de Feldman e Pentland (2000), entendem rotinas além do caráter repetitivo, trazendo à discussão a subjetividade através da capacidade de ação dos indivíduos. Seguindo essa linha de pensamento Pentland e Hærem (2015) indicam que a definição de rotinas organizacionais se ampliou, partindo de uma visão estreita e estereotipada e hoje abrangendo uma maior amplitude de fenômenos. De modo que o estudo atual sobre este tema envolve uma complexidade antes não vista nas visões tradicionais de, por exemplo, Cyert e March (1963) e Nelson e Winter (1982).

Nesta esteira, novos âmbitos associados às RO têm emergido e faz-se relevante entender como esse campo de investigação se comporta e de que modo contribui para o desenvolvimento do estudo das organizações. Diante do apresentado, a análise bibliométrica aqui apresentada tem por objetivo identificar o panorama atual sobre a literatura publicada em rotinas organizacionais, com o fim de revelar como este campo de estudo se caracteriza e quais as tendências e eventuais novas oportunidades para estudo.

\section{Metodologia}

Este trabalho se propôs a realizar um estudo bibliométrico que, de acordo com Okubo (1997), tem como objetivo mensurar a produção de pesquisa científica publicada já que desta maneira o conhecimento científico é divulgado. Ferreira e Alvarenga (2011) indicam que a bibliometria permite entender como se desenvolve e se comporta uma determinada área do conhecimento. De forma similar, Guedes e Borschiver (2005) indicam que a bibliometria pode ser entendida como uma ferramenta estatística, possibilitando a criação, análise e gestão de indicadores sobre determinado tema, contribuindo para o desenvolvimento de uma determinada área do conhecimento. Neste sentido, optou-se por uma pesquisa com abordagem quantitativa-descritiva, que tem como finalidade delinear ou analisar fenômenos, utilizando-se de mecanismos quantitativos para coletar sistematicamente dados ou amostras de populações (Marconi \& Lakatos, 2003). 
A investigação se iniciou com a seleção das bases de dados Scielo, Scopus e Web of Science. Estas bases de dados foram escolhidas por serem consideradas abrangentes e confiáveis. A base de dados Web of Science é tida como uma fonte de referência em qualidade para mais de 9 mil instituições acadêmicas, corporativas e governamentais (Clarivate Analitycs, 2020). Além disso, conta com mais de 50 anos de experiência na indexação de dados de forma precisa e consistente. Quanto à Scopus, a Elsevier (2020) indica que esta base se configura como o maior banco de dados de resumos e citações com revisão por pares da atualidade, o que garante amplitude no acesso à literatura relevante. A escolha pela base de dados Scielo deu-se em virtude de ela representar um importante mecanismo de acesso à produção científica nacional brasileira e de países de origem latina. Para Costas (2017) essas bases viabilizam a realização da pesquisa e, nesse sentido, são importantes recursos para o estudo.

A pesquisa nas bases de dados selecionadas foi realizada em dezembro de 2019, quando foram definidos os critérios de pesquisa como termos de busca, período temporal, tipo de documento e categoria. Nas bases de dados Scopus e Web of Science foi utilizado o termo de busca "organizational routines" considerado o período compreendido entre 2015 e 2019, tipo de documento "artigo" e categorias "business, management and accouting" para a primeira base de dados e "business" e "management" para a segunda. Nestes termos, a pesquisa retornou 119 artigos na base Scopus e 200 artigos na Web of Science. Para Scielo, utilizaram-se como palavras de busca "rotinas organizacionais", limitadas ao período de 2015 a 2019, tipo de documento "artigo" e subcategoria de pesquisa "gerenciamento". Na base Scielo, 5 artigos foram indicados como resultado de pesquisa.

Em seguida, os resultados de pesquisa foram extraídos de cada base de dados no formato de arquivo BibTeX e importados para o software Zotero, um sistema organizador em pesquisa científica. Neste programa, os artigos pesquisados em cada base foram organizados em "coleções" que, em conjunto, formam uma "biblioteca".

A análise, a partir deste momento, passou a ser feita no Zotero que, reunindo os 324 artigos, oriundos das 3 bases de dados, permitiu um filtro em que se restringiu a análise para documentos que contivessem em seu título os termos "routines" ou "rotinas", a fim de que incluíssem "organizational routines" ou "rotinas organizacionais". Em decorrência desta filtragem, uma nova "coleção" foi criada com um total de 103 documentos. Partindo destes 103 artigos, outra análise precisou ser feita, de modo a excluir artigos em duplicidade. Após a eliminação dos artigos em repetição, a amostra a ser analisada reduziu-se para a quantidade de 75 documentos (ver Tabela 1). 
Tabela 1: Quantidade de artigos a serem analisados após filtragens.

\begin{tabular}{l|l|l|l}
\hline Base de Dados & Scielo & Scopus & Web of Science \\
\hline Termos de busca & "rotinas organizacionais" & $\begin{array}{l}\text { "organizational } \\
\text { routines" }\end{array}$ & "organizational routines" \\
\hline Categorias de pesquisa & "gerenciamento" & $\begin{array}{l}\text { "business, } \\
\text { management accounting" }\end{array}$ & $\begin{array}{l}\text { "business" } \\
\text { "management" }\end{array}$ \\
\hline Quantidade de artigos & $\mathbf{5}$ & $\mathbf{1 1 9}$ & $\mathbf{2 0 0}$ \\
\hline Total de artigos & $\mathbf{3 2 4}$ & \\
\hline Software utilizado & Zotero & \\
\hline Filtro & "Routines" ou "Rotinas" no título \\
\hline Total de artigos & $\mathbf{1 0 3}$ & Eliminação de duplicidades \\
\hline Filtro & $\mathbf{7 5}$ & GoogleDocs \\
\hline Total de artigos & Análise de títulos, palavras-chaves e resumos/abstracts \\
\hline Software utilizado & $\mathbf{6 5}$ & \\
\hline Filtro & Total de artigos & & \\
\hline
\end{tabular}

Fonte: Autores.

A seguir, a lista de artigos foi estruturada em uma planilha eletrônica do GoogleDocs, e organizada por critérios como tipo do documento, ano de publicação, autor(es), título, periódico de publicação, identificador de objeto digital (DOI), endereço eletrônico, resumo/abstract, país de publicação, idioma, número de citações na base de dados respectiva, palavras-chave, dentre outros. Esta sistematização permitiu uma análise mais aprofundada de títulos, resumos, abstracts e palavras-chave a fim de investigar a aderência dos artigos selecionados ao interesse de pesquisa.

Neste momento, 4 artigos foram excluídos da amostra por não estarem disponíveis para leitura, ao mesmo tempo em que se eliminaram 6 artigos por não aderirem ao tema de Rotinas Organizacionais, tratando de ciência política ou rotinas familiares, por exemplo. Dessa maneira, a análise esteve focada em uma quantidade de 65 artigos, como observado na Tabela 1.

\section{Resultados e Discussão}

Nesta seção, inicia-se a análise dos resultados encontrados na pesquisa. Inicialmente, será apresentada uma análise bibliométrica sobre a amostra de artigos, em seguida, haverá a indicação dos temas de maior relevância e ocorrência no campo de rotinas organizacionais, bem como seus respectivos autores.

\subsection{Análise Bibliométrica}

O demonstrativo da quantidade de artigos publicados relacionados ao tema objeto de estudo deste trabalho é representado pela Figura 1. É possível identificar que o pico de publicação ocorreu em 2016 e 2017, quando foram publicados, respectivamente, 21 e 19 trabalhos relacionados ao tema. Diferentemente do que ocorreu em 2015, 2018 e 2019, quando a quantidade se manteve entre 9 e 8 artigos publicados por ano. A disparidade percebida entre 2016 e 2017, comparando-se com o período restante, pode ser explicada pelo fato de que, neste período, na edição de maio-junho de 2016, a Organization Science lançou uma edição especial direcionada para o tema de rotinas organizacionais. Este periódico, publicado pelo Institute for 
Operations Research and the Management Sciences, é reconhecido como de alta relevância para os estudos em teoria organizacional.

Figura 1. Quantidade de artigos publicados por ano.

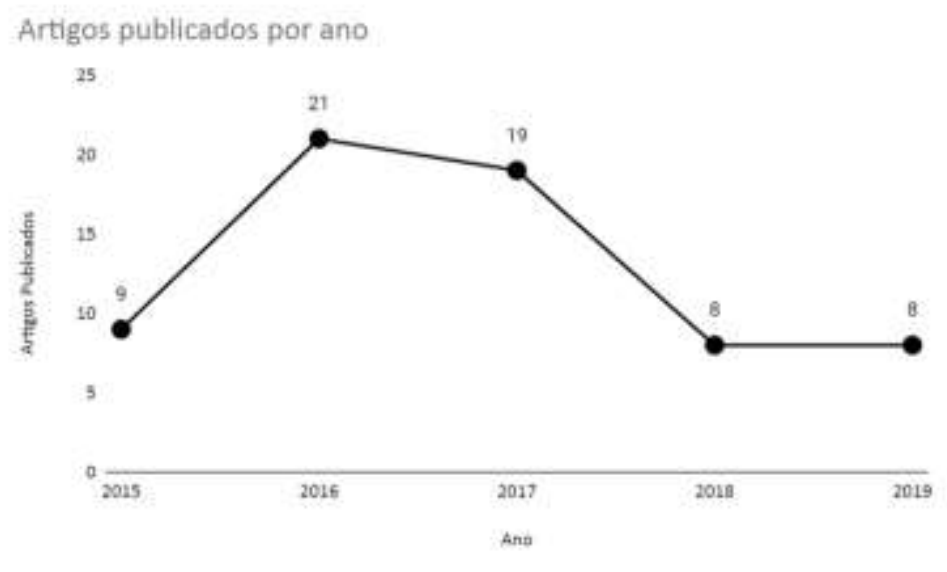

Fonte: Autores.

Dentre 45 periódicos identificados na pesquisa, a revista Organization Science se destacou com o maior número de artigos publicados no período, como demonstrado pela Figura 2. Este periódico esteve em primeiro lugar quanto à quantidade de publicações, na medida em que contou com 12 dos 65 artigos, ou seja, 18,46\%. Em segundo lugar, tem-se a Industrial and Corporate Change, com 3 artigos. Existem, ainda, outras 7 revistas com 2 publicações cada. Os 36 periódicos restantes contribuíram para o desenvolvimento do tema de rotinas organizacionais com a publicação de 1 artigo cada no período de 2015 a 2019.

Figura 2. Quantidade de artigos publicados por periódicos com maior ocorrência.

\section{Artigos publicados por periódicos de maior ocorrência}

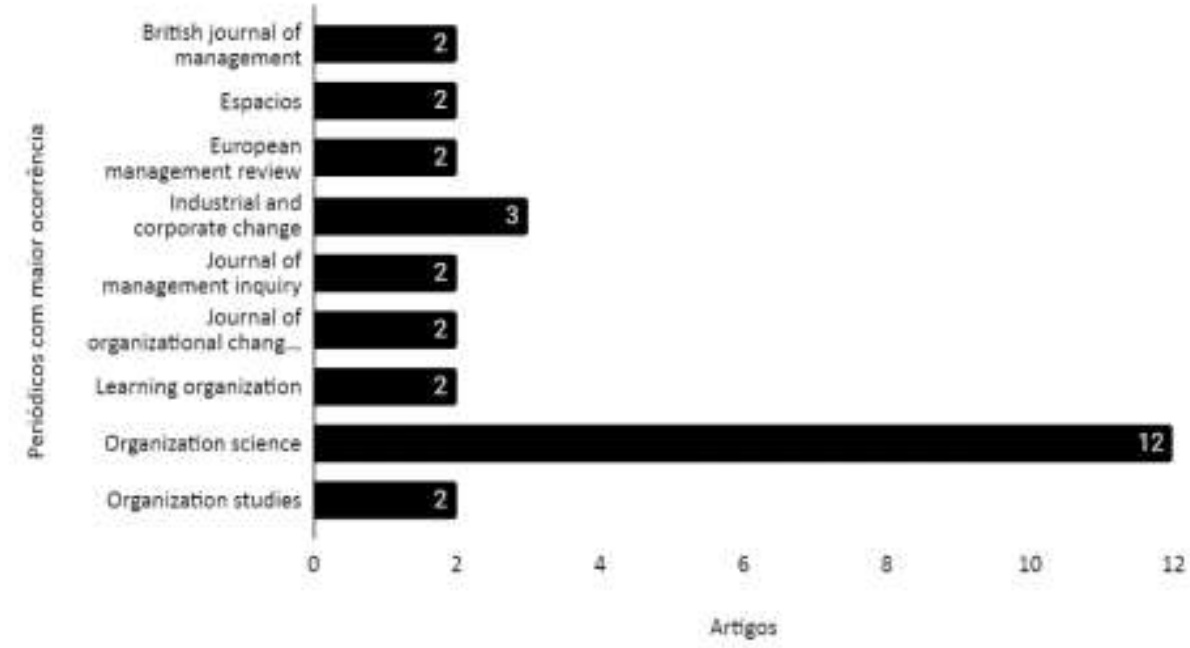

Fonte: Autores. 
Outro fator de análise bibliométrica em relação ao tema investigado, entre 2015 e 2019, recai sobre a identificação dos países de origem de cada publicação. A Figura 3 deixa evidente o panorama no qual tem-se que o tema de RO predomina entre países de origem e língua inglesa, dado que, dentre os 65 estudos, 34 são originários do Reino Unido e 19 têm origem nos Estados Unidos. De modo que estes países apoiaram o desenvolvimento dos estudos sobre rotinas organizacionais com cerca de $81,53 \%$ das publicações, fato que transparece a lacuna existente entre o conhecimento e pesquisa sobre o tema desenvolvido no exterior e no Brasil.

Figura 3. Quantidade de artigos publicados por país de origem.

\section{Artigos publicados por pais}

40

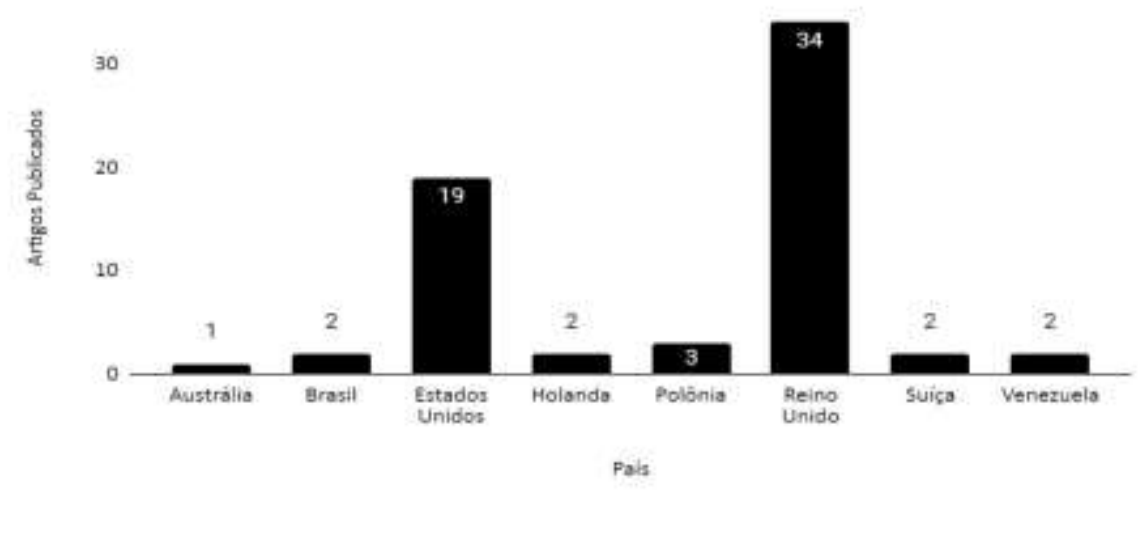

Fonte: Autores.

Como parte da análise realizada, o conjunto de palavras-chave dos 65 artigos estudados foi consolidado para a formação de uma nuvem de palavras com auxílio do software online wordcloud.com. Esta ferramenta permite visualizar os termos de maior ocorrência e mais associados ao assunto analisado. Essas palavras, apresentadas na Figura 4, demonstram que, dentro dos parâmetros analisados, o campo de estudo de rotinas organizacionais está alinhado com mais intensidade a temas como inovação, mudança, dinâmica, teoria, aprendizagem e gestão organizacional. 
Figura 4. Nuvem de palavras-chave relacionadas a rotinas organizacionais.

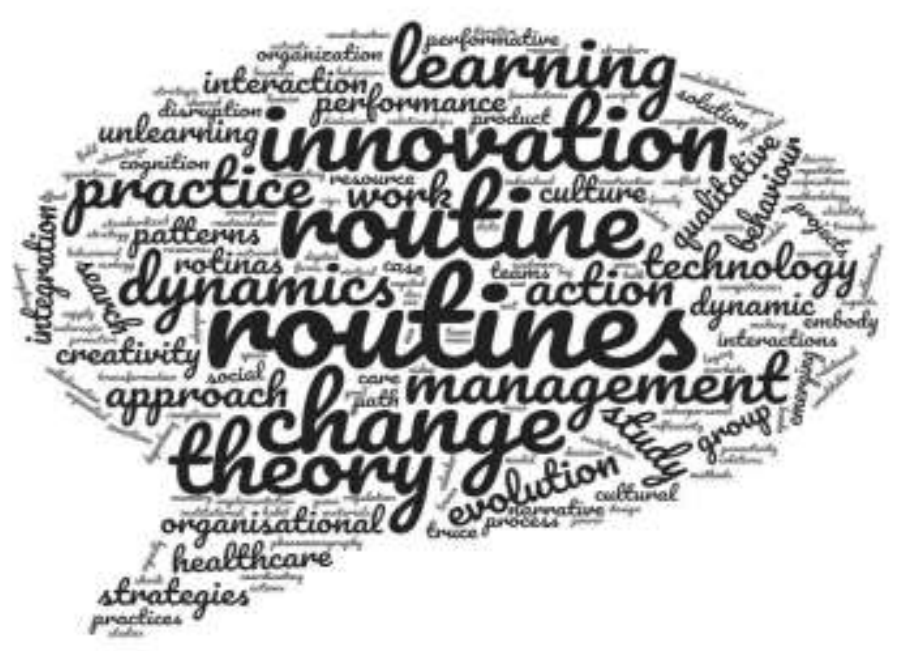

Fonte: Autores.

Após conhecer os temas mais prevalentes associados ao estudo de rotinas organizacionais, passa-se a analisar os artigos identificados como de maior relevância, aferida pela quantidade de citações entre as bases de dados. A Tabela 2 apresenta a relação dos 10 artigos mais citados, bem como indica o tema associado ao estudo de rotinas organizacionais e o tipo de estudo, teórico ou empírico.

Tabela 2: Relação de autores mais citados, temas e tipos de estudo.

\begin{tabular}{cccc} 
Autores & Citações & Tema & $\begin{array}{c}\text { Tipo de } \\
\text { estudo }\end{array}$ \\
\hline Wilhelm, Schlömer \& Maurer (2015) & 38 & Capacidades dinâmicas & Empírico \\
\hline Carayannis, Grigoroudis, Giudce, Della Peruta \& & 35 & Aprendizagem & Teórico \\
Sindakis (2017) & & Dinâmica das rotinas & Empírico \\
\hline Dittrich et al. (2016) & 30 & Dinâmica das rotinas & Empírico \\
\hline Sonenshein (2016) & 27 & Dinâmica das rotinas & Empírico \\
\hline Deken, Carlile, Berends \& Lauche (2016) & 23 & Mudança organizacional & Teórico \\
\hline Ki, Knudsen \& Becker (2016) & 23 & Dinâmica das rotinas & Teórico \\
\hline Bertels, Howard-Grenville \& Pek. (2016) & 23 & Dinâmica das rotinas & Empírico \\
\hline Berente Lyytinen, Yoo \& King (2016) & 22 & Dinâmica das rotinas & Empírico \\
\hline LeBaron, Christianson, Garrett \& Ilan (2016) & 21 & Dinâmica das rotinas & Empírico \\
\hline
\end{tabular}


Na investigação dos 10 artigos mais relevantes, nota-se uma predominância de estudos desenvolvidos empiricamente, fato que repercute a realidade observada na amostra de 65 artigos analisados. Além disso, em termos de temas associados, predomina a abordagem de dinâmica das rotinas organizacionais, como será detalhado na seção seguinte.

A análise a seguir se propõe a verificar o aspecto material produzido pelo campo de estudo em RO, ou seja, o que de fato foi estudado e alguns resultados obtidos.

\subsection{Análise do Campo de Estudo}

Ao analisar os dados percebe-se que a visão tradicional de rotinas organizacionais, em que são vistas como fontes de estabilidade, repetição e inércia, não aparecem nos estudos mais atuais. O campo de estudo recente tem apresentado foco em uma visão dinâmica, como previamente indicado pela Tabela 2, de modo que diversos artigos analisados tratam as RO como aspectos organizacionais ativos, e não estáticos (Bapuji, Hora, Saeed \& Turner, 2019; Salvato \& Rerup, 2017; Vough, Bindl \& Parker, 2017; Sargis-Roussel, Belmond \& Deltour, 2016; Mariano \& Casey, 2016; Habib \& Krohmer, 2016; Safavi \& Omidvar, 2016; Aroles \& Mclean, 2016; Deken et al., 2016; Berente et al., 2016; Dittrich et al., 2016; Kremser \& Schreyogg, 2016; Yamauchi \& Hiramoto, 2016; Lebaron et al., 2016; Bertels et al., 2016; Sonensheim, 2016; Angwin, Paroutis \& Connell, 2015; Kaplan, 2015). Essa visão sobre o tema resgata os estudos de Feldman (2000), que primeiramente tratou as ROs como elementos dinâmicos em uma organização, dissociando-se do entendimento anterior que tratava as rotinas como aspectos de necessária automatização.

A visão dinâmica para o estudo de rotinas organizacionais leva em conta a perspectiva de que são formadas por aspectos ostensivos e performativos, ou seja, a prática de uma rotina (performativo) é tão relevante quanto o entendimento abstrato de como ela deve ocorrer (ostensivo). Este panorama surgiu com os estudos de Feldman e Pentland (2003), indicando que a relação entre como uma rotina deve ser executada e sua própria execução permite uma dinamicidade organizacional capaz de gerar mudança. Safavi e Omidvar (2016), por exemplo, ampliam a discussão ao indicarem que a dinâmica de uma rotina é influenciada não apenas pela relação de controle e poder entre os aspectos ostensivo e performativo, mas também pela interação com o meio que a envolve.

Nessa esteira, outros temas de relevo associados às rotinas organizacionais são inovação, mudança e criatividade organizacional. Diversos autores (Bower, 2017; Arias-Peréz, Perdomo-Charry \& Castano-Ríos, 2017; Stańczyk-Hugiet, Piórkowska \& Stańczuk, 2017; Da Silva, Guevara, João, De Oliveira \& Fernandes, 2017; Patora-Wysocka, 2017; Elg, Ghauri, Child \& Collinson, 2017; Lin, Chen \& Su, 2017; Lindkvist, Bengtsson, Svensson \& Wahlstedt, 2016; Sele \& Grand, 2016; Cohendet \& Simon, 2016; Markowski \& Dabhilkar, 2016; Appiah, 2015) estudam as rotinas organizacionais como mecanismos indutores em um processo de inovação organizacional, possibilitando redução de inércia e inatividade. Sele e Grand (2016), por exemplo, em suas pesquisas identificam que o processo de inovação no contexto de uma RO pode ocorrer de três maneiras: através da criação de um novo efeito ou resultado inovador; da adaptação de uma rotina existente a uma nova situação; ou pelo desenvolvimento de uma nova rotina, consequentemente envolvendo criatividade e mudança em uma organização.

Além dos temas já citados, outro assunto foi discutido amplamente sobre rotinas organizacionais, a saber: aprendizagem organizacional. Diferentes autores (Müller, Buchele, Steil, Teza, Bonilla, Dandolini \& De Souza, 2017; Carayannis et al., 2017; Fiol \& O'connor, 2017a, 2017b; Cherman \& Rocha-Pinto, 2016) tratam deste tema indicando que as rotinas organizacionais são um elemento na apropriação de conhecimentos individuais e coletivos, influenciando no desenvolvimento da organização.

Mariano e Casey (2016) também tratam do tema indicando que a dinâmica de uma rotina e seu processo de variação pode ser explicado por ciclos inter relacionados de aprendizagem e esquecimento. Já Carayannis et al. (2017) relacionaram o estudo de ROs e artefatos, que, de acordo com Feldman e Pentland (2003), representam o aspecto material de uma rotina, como um ciclo de aprendizagem e obtenção de conhecimento que dá forma e realça a inteligência organizacional. 
Importante destacar que o estudo sobre capacidades dinâmicas também possui aproximação com o de rotinas organizacionais, como identificado nos estudos de Hilliard e Goldstein (2018), Mousavi, Bossink \& Van Vliet (2018), Wilhelm et al. (2015).

Apesar de o foco do campo de estudo em rotinas organizacionais dar destaque a suas características promotoras de mudança e inovação organizacional, estudos como o de Berente et al. (2016), Yi et al. (2016) e Guha (2015) reforçam a importância das RO como mecanismos estabilizadores da dinâmica e disrupção organizacional. Berente et al. (2016) adjetivam as rotinas como absorvedoras de impacto, na medida em que promovem a estabilidade organizacional necessária em contextos de desequilíbrio. De modo similar, Yi et al. (2016) indica que a inércia em rotinas organizacionais tem papel fundamental na estruturação de mudanças programadas, assim como permitem que seus efeitos ocorram em ritmo adequado.

\section{Considerações Finais}

A análise bibliométrica apresentada torna evidente como o campo de estudo em rotinas organizacionais se comporta no período de 2015 a 2019. Neste estrato temporal, percebe-se uma crescente em publicações sobre o tema nos anos de 2016 e 2017, havendo estabilização nos anos seguintes. O periódico com maior contribuição para o desenvolvimento do campo de RO foi o Organization Science, com 12 publicações. Além disso, em mais de 80\% dos casos, os artigos foram publicados em países de língua inglesa como Reino Unido e Estados Unidos.

O presente estudo pode avaliar, ainda, de que forma as rotinas organizacionais são estudadas, de modo que prevalece a visão moderna de que são mecanismos fluidos, voláteis e dinâmicos, com a possibilidade de atuarem como indutores de mudança e inovação organizacional. Há, portanto, uma tendência para rompimento com o antigo entendimento de que a rotinização em uma organização seria fator de inércia e inflexibilidade. Apesar disso, percebe-se que as rotinas organizacionais ainda desempenham papel fundamental como meio de estabilização em uma organização, em especial em momentos de turbulência (Berente et al., 2016; Yi et al., 2016; Guha, 2015).

Esta análise contribui para o desenvolvimento da área de estudo pois permite a identificação dos temas e vieses para o campo de ROs. No período de 2015 a 2019, diversos temas foram apontados como de interesse como dinâmica das rotinas, inovação, mudança, aprendizagem, capacidades dinâmicas e estabilidade organizacional. Outrossim, esta pesquisa pode demonstrar uma lacuna existente entre o desenvolvimento do tema de rotinas organizacionais no Brasil e no exterior, evidenciada pela baixa produção científica publicada por autores e periódicos brasileiros.

Por se tratar de um estudo bibliométrico cujo objetivo é realizar uma análise ampla do campo, entende-se a necessidade de novas pesquisas que possam trazer profundidade a cada tema relacionado a ROs aqui identificados. Além disso, é interessante avaliar com maior precisão, com uso de softwares de análise estatística, as potenciais interfaces e correlações entre estudos da amostra delimitada.

Desse modo, sugestiona-se que pesquisas futuras podem afunilar o escopo de investigação, visando o aprofundamento do estudo sobre rotinas organizacionais. Indica-se que, para isso, seja realizada uma revisão sistemática de literatura com seus métodos próprios e com foco em uma questão específica de pesquisa. Além disso, seria interesse ampliar o período temporal de análise a fim de avaliar a frequência de publicações sobre o tema e verificar a relação entre os estudos teóricos e empíricos sobre rotinas organizacionais. Por fim, orienta-se que sejam desenvolvidos estudos sobre o tema no contexto brasileiro, de modo a preencher a lacuna existente entre a produção nacional e a estrangeira. 


\section{Referências}

Angwin, D. N., Paroutis, S., \& Connell, R. (2015). Why good things Don't happen: the micro-foundations of routines in the M\&A process. Journal Of Business Research, 68(6), p.1367-1381. https://www.sciencedirect.com/science/article/abs/pii/S0148296314004226?via\%3Dihub. doi: https://doi.org/10.1016/j.jbusres.2014.12.007.

Appiah, G. (2015). How creative are SMEs? An organizational routines' perspective. International Journal Of Technology Management \& Sustainable Development, 14(2), p.141-157. https://www-scopus.ez19.periodicos.capes.gov.br/record/display.uri?eid=2-s2.0-84942318299\&origin=resultslist\&sort=plf$\mathrm{f} \& \mathrm{src}=\mathrm{s} \& \mathrm{sid}=\mathrm{bd} 6 \mathrm{ff} 7 \mathrm{e} 61648 \mathrm{a} 40 \mathrm{fc} 6 \mathrm{cc} 13 \mathrm{e} 1 \mathrm{a} 2 \mathrm{ceaccd} \& \mathrm{sot}=$ autdocs $\& \mathrm{sdt}=$ autdocs $\& \mathrm{sl}=18 \& \mathrm{~s}=\mathrm{AU}-\mathrm{ID} \% 2856798338800 \% 29 \& \mathrm{relpos}=2 \& \mathrm{citeCnt}=1 \& \mathrm{searchTerm}=$. doi: https://doi.org/10.1386/tmsd.14.2.141_1

Arias-Pérez, J., Perdomo-Charry, G., \& Castano-Ríos, C. (2017). Not-invented-here Syndrome And Innovation Performance: The Confounding Effect Of Innovation Capabilities As Organisational Routines In Service Firms. International Journal Of Innovation Management, 21(01). https://www.worldscientific.com/doi/abs/10.1142/S1363919617500360. doi: https://doi.org/10.1142/S1363919617500360.

Aroles, J., \& Mc Lean, C. (2016). Rethinking Stability and Change in the Study of Organizational Routines: Difference and Repetition in a Newspaper-Printing Factory. Organization Science. 7(3), 535-550. https://pubsonline.informs.org/doi/abs/10.1287/orsc.2015.1035. doi: https://doi.org/10.1287/orsc.2015.1035.

Bapuji, H., Hora, M., Saeed, A., \& Turner, S. (2019). How Understanding-Based Redesign Influences the Pattern of Actions and Effectiveness of Routines. Journal Of Management, 45(5), 2132-2162. https://journals.sagepub.com/doi/abs/10.1177/0149206317744251. Doi: https://doi.org/10.1177/0149206317744251.

Becker, M. C. (2008). The past, present and future of organizational routines: Introduction to the handbook of organizational routines. In: Handbook of Organizational Routines. Becker M. C. (ed.). Cheltenham UK and Northampton MA: Edward Elgar, 2008.

Berente, N., Lyytinen, K., Yoo, Y., \& King, J. L. (2016). Routines as Shock Absorbers During Organizational Transformation: Integration, Control, and NASA's Enterprise Information System. Organization Science. 7(3), 551-572. https://pubsonline.informs.org/doi/abs/10.1287/orsc.2016.1046. Doi: https://doi.org/10.1287/orsc.2016.1046.

Bertels, S., Howard-Grenville, J., \& Pek, S. (2016). Cultural Molding, Shielding, and Shoring at Oilco: The Role of Culture in the Integration of Routines. Organization Science, 27(3), 573-593. https://pubsonline.informs.org/doi/10.1287/orsc.2016.1052. Doi: https://doi.org/10.1287/orsc.2016.1052.

Broman, S. L. S., Ruas, R. L., \& Rocha-pinto, S. R. (2019). A Construção de Competências Coletivas na Dinâmica das Rotinas Orçamentárias. Cadernos EBAPE.BR, 17(Edição Especial), 871-885. https://www.scielo.br/j/cebape/a/pRr5jpKNgyw36FVdjVwM75y/?format=pdf\&lang=pt. Doi: http://dx.doi.org/10.1590/1679-395174729

Bower, J. (2017). Whitbread: routines and resource building on the path from brewer to retailer. Management \& Organizational History, 13(1), 1-23. Recuperado de: https://www.tandfonline.com/doi/abs/10.1080/17449359.2017.1408471?journalCode=rmor20. Doi: https://doi.org/10.1080/17449359.2017.1408471.

Carayannis, E. G., Grigoroudis, E., Giudice, M., Della Peruta, M. R., \& Sindakis, S. (2017). An exploration of contemporary organizational artifacts and routines in a sustainable excellence context. Journal Of Knowledge Management, 21(1), 35-56. https://www.emerald.com/insight/content/doi/10.1108/JKM-10-20150366/full/html.

Cherman, A., \& Rocha-Pinto, S. R. (2016). Valuing of knowledge in organizations and its embedding into organizational practices and routines. Review Of Business Management, 18(61), 416-435. http://www.scielo.br/scielo.php?pid=S1806-48922016000300416\&script=sci_arttext\&tlng=en. Doi: 10.7819/rbgn.v18i61.2966.

Cohendet, P. S., \& Simon, L. O. (2016). Always Playable: Recombining Routines for Creative Efficiency at Ubisoft Montreal's Video Game Studio. Organization Science, 27(3), 614-632. https://pubsonline.informs.org/doi/10.1287/orsc.2016.1062. Doi: https://doi.org/10.1287/orsc.2016.1062.

Costas, R. (2017). Discussões gerais sobre as características mais relevantes de infraestruturas de pesquisa para a cientometria. In: Bibliometria e Cientometria no Brasil: infraestrutura para avaliação da pesquisa científica na Era do Big Data, 19-42. Recuperado de: https://doi.org/10.11606/9788572051705.

Clarivate Analytics. (2020). Web of Science: Confident research begins here. https://clarivate.ez19.periodicos.capes.gov.br/webofsciencegroup/solutions/webof-science/?utm_source=webofsciencegroup\&utm_medium=website\&utm_campaign=for_research.

Cyert, R. M., \& March, J. G. (1963). A Behavioral Theory of the Firm. Prentice Hall: Englewood Cliffs, NJ, 1963.

Silva, L. F., Guevara, A. J., João, B. N., De Oliveira, P. S. G., \& Fernandes, K. R. (2017). Evolutionary theory framework to understand change in organizational routines. Espacios $38(24)$.

https://www.researchgate.net/publication/317045310_Evolutionary_theory_framework_to_understand_change_in_organizational_routines.

Deken, F., Carlile, P. R., Berends, H., \& Lauche, K. (2016). Generating Novelty Through Interdependent Routines: A Process Model of Routine Work. Organization Science, 27(3), 659-677. https://pubsonline.informs.org/doi/10.1287/orsc.2016.1051. Doi: https://doi.org/10.1287/orsc.2016.1051.

Dittrich, K., Guérard, S., \& Seidl, D. (2016). Talking About Routines: The Role of Reflective Talk in Routine Change. Organization Science. 7(3), 678-697. https://pubsonline.informs.org/doi/abs/10.1287/orsc.2015.1024. Doi: https://doi.org/10.1287/orsc.2015.1024.

Elg, U., Ghauri, P. N., Child, J., \& Collinson, S. (2017). MNE microfoundations and routines for building a legitimate and sustainable position in emerging markets. Journal Of Organizational Behavior, 38(9), 1320-1337. Recuperado de: https://onlinelibrary.wiley.com/doi/abs/10.1002/job.2214. Doi: https://doi.org/10.1002/job.2214.

Elsevier. (2020). Scopus. https://www.elsevier.com/pt-br/solutions/scopus.

Feldman, M. S. (2000). Organizational routines as a source of continuous change. Organization Science. 11(6), 611-629. https://pubsonline.informs.org/doi/abs/10.1287/orsc.11.6.611.12529. Doi: https://doi.org/10.1287/orsc.11.6.611.12529. 
Feldman, M. S., \& Pentland, B. T. (2003) Reconceptualizing Organizational Routines as a Source of Flexibility and Change. Administrative Science Quarterly, 48(1), 94-118. http://journals.sagepub.com/doi/pdf/10.2307/3556620. Doi: https://doi.org/10.2307/3556620.

Feldman, M. S., Pentland, T. B., D’adderio, L., \& Lazaric, N. (2016). Beyond Routines as Things: Introduction to the Special Issue on Routine Dynamics. Organization Science. 7(3), 505-513. Recuperado de: https://pubsonline.informs.org/doi/abs/10.1287/orsc.2016.1070. Doi: https://doi.org/10.1287/orsc.2016.1070.

Ferreira Araújo, R., \& Alvarenga, L. (2011). A bibliometria na pesquisa científica da pós-graduação brasileira de 1987 a 2007 . Encontros Bibli: revista eletrônica de biblioteconomia e ciência da informação. 16(31). https://periodicos.ufsc.br/index.php/eb/article/view/1518-2924.2011v16n31p51/17757. Doi: 10.5007/15182924.2011v16n31p51.

Figueira, E. C. S., \& Rocha-Pinto, S. R. (2018). Mapa ou território? A experiência de consultores na identificação de rotinas organizacionais. Revista de Administração FACES, 17(4), 108-128. http://revista.fumec.br/index.php/facesp/article/view/5921. Doi: https://doi.org/10.21714/19846975FACES2018V17N4ART5921

Fiol, M., \& O'connor, E. (2017a) Unlearning established organizational routines - Part I. The Learning Organization, 24(1), 13-29. https://www.emerald.com/insight/content/doi/10.1108/TLO-09-2016-0056/full/html. Doi: https://doi.org/10.1108/TLO-09-2016-0056.

Fiol, C. M., \& O’connor, E. J. (2017b). Unlearning established organizational routines - Part II. The Learning Organization, 24(2), 82-92. https://www.emerald.com/insight/content/doi/10.1108/TLO-09-2016-0063/full/html. Doi: https://doi.org/10.1108/TLO-09-2016-0063.

Gersick, C. J. G., \& J. Hackman, J. R. (1990). Habitual Routines in Task-Performing Groups. Organizational Behavior and Human Decision Processes. 47, 6597. http://studylib.net/doc/18750942/habitual-routines-in-task-performing-groups.

Guedes, V. L. S., \& Borschiver, S. (2005). Bibliometria: uma ferramenta estatística para a gestão da informação e do conhecimento, em sistemas de informação, de comunicação e de avaliação científica e tecnológica. In: Encontro nacional de ciências da informação, 6., Salvador/BA, junho de 2005. http://www.cinformanteriores.ufba.br/vi_anais/docs/VaniaLSGuedes.pdf.

Guha, M. (2015). Routine responses to disruption of routines. International Journal Of Learning And Change, 8(2), 136-161. https://www.researchgate.net/publication/289584436_Routine_responses_to_disruption_of_routines. Doi: 10.1504/IJLC.2015.074065.

Habib, J., \& Krohmer, C. (2016). Balanced or unbalanced routines: the case of two routines dynamics in a French hospital. Journal Of Organizational Change Management, 29(4), 508-528. https:/www.emerald.com/insight/content/doi/10.1108/JOCM-11-2015-0212/full/html. Doi: https://doi.org/10.1108/JOCM-112015-0212.

Hilliard, R., \& Goldstein, D. (2018). Identifying and measuring dynamic capability using search routines. Strategic Organization, 17(2), 210-240. https://journals.sagepub.com/doi/abs/10.1177/1476127018755001. Doi: https://doi.org/10.1177/1476127018755001.

Kaplan, S. (2015). Truce Breaking and Remaking: The CEO's Role in Changing Organizational Routines. Cognition and Strategy, 32, 1-45. https://www.emerald.com/insight/content/doi/10.1108/S0742-332220150000032001/full/html. Doi: https://doi.org/10.1108/S0742-332220150000032001.

Kremser, W., \& Schreyögg, G. (2016). The Dynamics of Interrelated Routines: Introducing the Cluster Level. Organization Science, 27(3), 698-721. https://pubsonline.informs.org/doi/abs/10.1287/orsc.2015.1042?af=R. doi: https://doi.org/10.1287/orsc.2015.1042.

Lebaron, C., Christianson, M. K., Garret, L., \& Ilan, R. (2016). Coordinating Flexible Performance During Everyday Work: An Ethnomethodological Study of Handoff Routines. Organization Science, 27(3), 514-534. https://pubsonline.informs.org/doi/10.1287/orsc.2015.1043. Doi: https://doi.org/10.1287/orsc.2015.1043.

Lin, H., Chen, M., \& Su, J. (2017). How management innovations are successfully implemented? An organizational routines' perspective. Journal Of Organizational Change Management, 30(4), 456-486.: https://www.emerald.com/insight/content/doi/10.1108/JOCM-07-2016-0124/full/html. Doi: https://doi.org/10.1108/JOCM-07-2016-0124.

Lindkvist, L., Bengtsson, M., Svensson, D., \& Wahlstedt, L. (2016). Replacing old routines: how Ericsson software developers and managers learned to become Agile. Industrial And Corporate Change, 26(4), 571-591. https://academic.oup.com/icc/article-abstract/26/4/571/2907944?redirectedFrom=fulltext. Doi: https://doi.org/10.1093/icc/dtw038.

Marconi, M. A., \& Lakatos, E. M. (2003). Fundamentos de metodologia científica. 5. ed. São Paulo: Atlas.

Mariano, S., \& Casey, A. (2016). The Dynamics of Organizational Routines in a Startup: The Ereda Model. European Management Review, 13(4), 251-274. https://onlinelibrary.wiley.com/doi/10.1111/emre.12080. Doi: https://doi.org/10.1111/emre.12080.

Markowski, P., \& Dabhilkar, M. (2016). Collaboration for continuous innovation: routines for knowledge integration in healthcare. International Journal Of Technology Management, 71(3/4), 212-231. https://www.inderscienceonline.com/doi/abs/10.1504/IJTM.2016.078569. Doi: https://doi.org/10.1504/IJTM.2016.078569.

Melo, G. T., \& Machado, A. G. C. (2020). Aprendizagem, strategining e rotinas organizacionais no processo de desenvolvimento de produtos sob a perspectiva das capacidades dinâmicas. Revista de $21(5), \quad$ administração https://www.scielo.br/j/ram/a/YqZM7bpKzx3m4BcrnMxYGQp/?format=pdf\&lang=pt. Doi: 10.1590/1678-6971/eRAMR200153.

Mousavi, S., Bossink, B., \& Van Vliet, M. (2018). Dynamic capabilities and organizational routines for managing innovation towards sustainability. Journal Of Cleaner Production, 203, 224-239. https://www.sciencedirect.com/science/article/pii/S0959652618325642. Doi: https://doi.org/10.1016/j.jclepro.2018.08.215.

Müller, I. R. F., Buchele, G. T., Steil, A. V., Teza, P., Bonilla, M. A. M., Dandolini, G. A., \& De Souza, J. A. (2017). Organizational learning and unlearning in footwear companies: A study on osed 38 (16). https://www.researchgate.net/publication/316212689_Organizational_learning_and_unlearning_in_footwear_companies_A_study_based_on_organizational_r outines. 
Nascimento, C. C. L., Silva, B. V. C., Oliveira, J. G.C., Nascimento, M. F. S., Gomes, Y., \& Moreira, L. C.S. (2020).Educação permanente em sala de imunização: elaboração de manual de normas e Rotinas. Research, Society and Development, 9(8), 1-12. https://rsdjournal.org/index.php/rsd/article/view/5601/4634. Doi: http://dx.doi.org/10.33448/rsd.

Nelson, R. R., \& Winter, S. G. (1982). An Evolutionary Theory of Economic Change. Harvard University Press: Cambridge, MA, 1982.

Okubo, Y. (1997). Bibliometric Indicators and Analysis of Research Systems: Methods and Examples, OECD Science, Technology and Industry Working Papers, OECD Publishing.Systems. Recuperado de: http://dx.doi.org/10.1787/208277770603.

Parmigiani, A., \& Howard-Grenville, J. (2011). Routines revisited: Exploring the capabilities and practice perspectives. The Academy of Management Annals 5(1), 413-453. http://www.tandfonline.com/doi/abs/10.1080/19416520.2011.589143. Doi: https://doi.org/10.1080/19416520.2011.589143.

Patora-Wysocka, Z. (2017). Beyond Stability vs. Change Dilemma: Everyday Practices and Routines as Sources of Organizational Life. Entrepreneurial Business And Economics Review, 5(1), 201-212. https://eber.uek.krakow.pl/index.php/eber/article/view/256. Doi: https://doi.org/10.15678/EBER.2017.050112.

Pentland, B. T., \& Feldman, M. S. (2005). Organizational Routines as Units of Analysis. Industrial and Corporate Change. 14(5), 793-815. https://academic.oup.com/icc/article/14/5/793/656208. Doi: https://doi.org/10.1093/icc/dth070.

Pentland, B. T., \& Feldman, M. S. (2008). Issues in empirical studies of organizational routines. In: Becker M. C. (ed.) Handbook of Organizational Routines. Cheltenham UK and Northampton MA: Edward Elgar.

Pentland, B. T., \& Haerem, T. (2015). Organizational Routines as Patterns of Action: Implications for Organizational Behavior. Annual Review Of Organizational Psychology And Organizational Behavior, 2(1), 465-487. https://www.annualreviews.org/doi/abs/10.1146/annurev-orgpsych-032414-111412. Doi: https://doi.org/10.1146/annurev-orgpsych-032414-111412.

Safavi, M., \& Omidvar, O. (2016). Resist or Comply: The Power Dynamics of Organizational Routines during Mergers. British Journal Of Management, 27(3), 550-566. https://onlinelibrary.wiley.com/doi/full/10.1111/1467-8551.12167. Doi: https://doi.org/10.1111/1467-8551.12167.

Salvato, C., \& Rerup, C. (2017). Routine Regulation: Balancing Conflicting Goals in Organizational Routines. Administrative Science Quarterly, 63(1), 170209. https://journals.sagepub.com/doi/full/10.1177/0001839217707738. Doi: https://doi.org/10.1177/0001839217707738.

Sargis-Roussel, C., Belmondo, C., \& Deltour, F. (2016). Bringing People Back in: How Group Internal Social Capital Influences Routines' Emergence. European Management Review, 14(1), 101-112. https://onlinelibrary.wiley.com/doi/10.1111/emre.12100. Doi: https://doi.org/10.1111/emre.12100.

Schröder, A., \& Geiger, D. (2016). Unravelling the Motor of Patterning Work: Toward an Understanding of the Microlevel Dynamics of Standardization and Flexibility. Organization Science. 7(3), 633-658. https://pubsonline.informs.org/doi/citedby/10.1287/orsc.2016.1055. Doi: https://doi.org/10.1287/orsc.2016.1055.

Sele, K., \& Grand, S. (2016). Unpacking the Dynamics of Ecologies of Routines: Mediators and Their Generative Effects in Routine Interactions. Organization Science, 27(3), 722-738. Recuperado de: https://pubsonline.informs.org/doi/10.1287/orsc.2015.1031. Doi: https://doi.org/10.1287/orsc.2015.1031.

Sonenshein, S. (2016). Routines and Creativity: From Dualism to Duality. Organization Science, 27(3), 739-758. Recuperado de: https://pubsonline.informs.org/doi/10.1287/orsc.2016.1044. Doi: https://doi.org/10.1287/orsc.2016.1044.

Stańczyk-Hugiet, E., Piórkowska, K., \& Stańcqyk, S. (2017). Organizational routines and context alteration: a reconciliation. Argumenta Oeconomica, 2(39), 341-372. Recuperado de: https://publons.com/publon/3334756/.

Vough, H. C., Bindl, U. K., \& Parker, S. K. (2017). Proactivity routines: The role of social processes in how employees self-initiate change. Human Relations, 70(10), 1191-1216. Recuperado de: https://journals.sagepub.com/doi/10.1177/0018726716686819. Doi: https://doi.org/10.1177/0018726716686819.

Wilhelm, H., Schlömer, M., \& Maurer, I. (2015). How Dynamic Capabilities Affect the Effectiveness and Efficiency of Operating Routines under High and Low Levels of Environmental Dynamism. British Journal Of Management, 26(2), 327-345. Recuperado de: https://onlinelibrary.wiley.com/doi/10.1111/14678551.12085. Doi: https://doi.org/10.1111/1467-8551.12085.

Yamauchi, Y., \& Hiramoto, T. (2016). Reflexivity of Routines: An Ethnomethodological Investigation of Initial Service Encounters at Sushi Bars in Tokyo. Organization Studies, 37(10), 1473-1499. Recuperado de: https://journals.sagepub.com/doi/10.1177/0170840616634125. Doi: https://doi.org/10.1177/0170840616634125.

Yi, S., Knudsen, T., \& Becker, M. C. (2016). Inertia in Routines: A Hidden Source of Organizational Variation. Organization Science, 27(3), 782-800. Recuperado de: https://pubsonline.informs.org/doi/10.1287/orsc.2016.1059. Doi: https://doi.org/10.1287/orsc.2016.1059. 